\title{
POST KALA-AZAR DERMAL LEISHMANIASIS WITH ULCERATION ON FOOT: AN ATYPICAL CASE PRESENTATION SUCCESSFULLY TREATED WITH MILTEFOSINE
}

\author{
${ }^{1}$ Vidyanand Rabi Das, ${ }^{2}$ Dharmendra Singh, \\ ${ }^{3}$ Krishna Murti, ${ }^{2}$ Sushmita Das, ${ }^{2}$ Pradeep Das and ${ }^{1}$ Krishna Pandey \\ ${ }^{1}$ Department of Clinical Medicine, \\ ${ }^{2}$ Department of Molecular Biology, \\ Rajendra Memorial Research Institute of Medical Sciences (Indian Council of Medical Research), \\ Agamkuan, Patna, Bihar, India \\ ${ }^{3}$ Department of Pharmacy Practice (National Institute of Pharmacy and Education Research), Hajipur, Bihar, India
}

Received 2014-02-28; Revised 2014-03-17; Accepted 2014-04-02

\begin{abstract}
Visceral Leishmaniasis (VL) or Kala-Azar (KA) is highly endemic in the Indian subcontinent particularly in Bihar. Post Kala-azar Dermal Leishmaniasis (PKDL) is a known reservoir for leishmania parasites for transmission of VL. The kala-azar elimination program was launched by the government of three countries of the Indian subcontinent in the year 2005, which aims to eliminate kala-azar by 2015 and PKDL by 2018. PKDL diagnosis and management has not been addressed properly. In this case report we have described a case of PKDL with ulcer and atypical presentation who was subsequently diagnosed by demonstration of Leishmania donovani bodies in the skin snips through microscopy, kinetoplast-DNA nested PCR and clinically. The patient was treated with miltefosine capsules in the dose of $50 \mathrm{mg}$ twicea-day for twelve-weeks and responded completely. This case report assumes great importance as prompt diagnosis and treatment of PKDL is essential in the kala-azar elimination program.
\end{abstract}

Keywords: PKDL, VL, Miltefosine, Ulcer, Kala-Azar Elimination Program

\section{INTRODUCTION}

Post Kala-azar Dermal Leishmaniasis (PKDL), caused by Leishmania donovani is a dermatosis usually occurring as a sequel of Visceral Leishmaniasis (VL). It is mainly reported from India (5-10\%), Sudan (50\%), Bangladesh and Nepal after successful treatment for VL (Zijlstra et al. 2003). In India, it was first described by (Brahmachari, 1992). About $10-20 \%$ cases have been reported to manifest without previous history of VL. This may be due to asymptomatic or subclinical infection that do not manifest as VL but had infection in the past (Das et al.,
2012). The incubation period of PKDL after VL is usually 0-6 months in Sudan and 6 months to several years in India. PKDL manifestation usually starts with macular lesions, progressing to papular and nodular types at the advanced stage (Zijlstra et al., 2000). The kala-azar elimination program aims to eliminate VL by 2015 and PKDL by 2018 from the Indian subcontinent (Verma et al., 2013). For this aim successful diagnosis and treatment of VL and PKDL is of utmost importance. In this report, we present a unique case of PKDL from Bihar, India with ulcer on foot as an atypical manifestation of PKDL which was successfully treated with miltefosine.

Corresponding Author: Krishna Pandey, Department of Clinical Medicine, Rajendra Memorial Research Institute of Medical Sciences, (Indian Council of Medical Research) Agamkuan, Patna-800 007, Bihar, India Tel: +919431042119 Fax: +916122634379 


\section{CASE REPORT}

A 17-year-old unmarried female patient, hailing from a VL endemic village of Bihar, with de-pigmented macular, papular and nodular lesions visited the outpatient-clinic of Rajendra Memorial Research Institute of Medical Sciences, Patna, Bihar, India. The patient had history of VL seven years back and was cured (clinically and parasitologically) with Sodium Stibogluconate (SAG) intra-muscular injection in the dose of $20 \mathrm{mg} \mathrm{kg}^{-1}$ body weight for 30 days. But after two years of VL treatment, de-pigmented patches started developing on the face, gradually spreading to other parts of body and progressing to papular and nodular lesions.

Macular lesions were present all over the body, more marked on the arms (Fig. 1A) and legs and the papular lesions were more marked on chin. Moreover, an ulcer (size $5 \times 4 \mathrm{~cm})$ was present on dorsum of right foot that emerged from a warty elevated nodule like appearance over a period of two months. Ulcer margin was irregular and islands of intact skin patches were present within the area of ulcer but without erythema. Granulation of lesional tissue was also present on lateral margin of ulcer (Fig. 2A). There were multiple nodular lesions on postero-medial aspect and tendo-achilles of the same foot (Fig. 3A). The upper most nodules, located on the posterior aspect of lower one-third of right leg, had a tendency to ulcerate along with the hyperemic surrounding skin. The nodules present on other sites of the same foot also had tendency to ulcerate. Furthermore, it was disclosed by the patient that prior to visiting this centre, she had been treated with systemic antibiotics and local anti-fungal and anti-biotic ointments without any improvement in the lesion profile. After consultation with a dermatologist, leprosy was excluded clinically based on non-anesthetic nature of the lesions and no thickening of peripheral ulnar nerves. Acid Fast Bacilli (AFB) from skin snips and nasal smears were also found negative by ZiehlNeelsen staining. Eczematous dermatitis, Pityriasis and other fungal infections were also ruled out.

Clinical diagnosis of PKDL with ulcer was made and the patient was admitted in the ward for further investigations and treatment. Signed informed consent was obtained from the patient. The systemic examination revealed-body weight $41 \mathrm{~kg}$, height $160 \mathrm{~cm}$, body mass index $16 \mathrm{~kg} / \mathrm{m}^{2}$, pulse $82 / \mathrm{min}$, BP $118 / 76 \mathrm{mmHg}$ and no findings of anemia, dehydration, hepatosplenomegaly, lymphadenopathy and cardiac abnormality. The biochemical and pathological parameters were recorded as: Total protein $6.75 \mathrm{~g} \mathrm{dL}^{-1}$, albumin $3.39 \mathrm{~g} \mathrm{dL}^{-1}$, globulin $3.36 \mathrm{~g} \mathrm{dL}^{-1}$, albumin: Globulin ratio $1: 1$, serum biluribin $0.92 \mathrm{mg} \mathrm{dL}^{-1}$, blood urea $18 \mathrm{mg} \mathrm{dL}^{-1}$, serum creatinine $0.59 \mathrm{mg} \mathrm{dL}^{-1}$, fasting blood sugar $86 \mathrm{mg} \mathrm{dL}^{-1}$, Aspartate Transaminase (AST) 24.1U/L, Alanine Transaminase (ALT) 17.6U/L, alkaline phosphatase 196U/L, Red Blood Cells (RBCs) count 4.02 millions $/ \mathrm{mm}^{3}$, white blood cell count $8900 / \mathrm{mm}^{3}$, neutrophil 59\%, lymphocyte $30 \%$, monocyte $5 \%$, eosinophil $6 \%$, basophil $0 \%$, platelets 156000 per $\mathrm{mm}^{3}$ and $\mathrm{Hb} 8.2 \mathrm{gm} / \mathrm{dL}$. HIV, hepatitis $\mathrm{B}$ and $\mathrm{C}$ were negative. Pregnancy test was negative in urine.

rK39 strip test (Kala-azar Detect ${ }^{\mathrm{TM}}$, Rapid Test, InBios International, Seattle, WA, USA) was positive with serum according to the manufacturer's instructions (Salotra et al., 2001). Microscopic examination of skin snips collected from different sites of macules, papules, nodules, ulcerated area and surrounding ulcer, revealed presence of Leishmania donovani bodies confirming it to be a case of PKDL. The result was confirmed by kinetoplast-DNA (k-DNA) nested-Polymerase Chain Reaction (n-PCR) for Leishmania donovani from blood and skin snips (Sreenivas et al., 2004).

After clinical, parasitological and molecular confirmation, the patient was treated with oral miltefosine capsules in the dose of $50 \mathrm{mg}$ twice-a-day for twelve-weeks with an advice to come for follow-up every four weeks. On follow-up visit after four-weeks of treatment, she was re-admitted for clinical, biochemical, pathological, parasitological and molecular assessment. Clinically the ulcer was not fully healed. Microscopic examination of skin snips was again found positive for LD bodies and by k-DNA nested-PCR. After eightweeks, multiple nodular lesions over the right foot started reducing in size. The de-pigmented patches also started disappearing (Fig. 1B) and nodules on face completely disappeared too.

On 3rd follow-up visit after twelve-weeks, the patient was found parasitologically cured, but the lesions were still persistent in diminished form. Ulcers were completely healed, though healed scar was present on the ulcerated area (Fig. 2B and 3B). The main adverse events included loose motions and vomiting which was easily controlled with treatment. There was also elevation of alanine and aspartate transaminase in the 1 st follow-up visit but came down to normal in the 2 nd follow-up. 
Vidyanand Rabi Das et al. / American Journal of Infectious Diseases 10 (2): 50-55, 2014

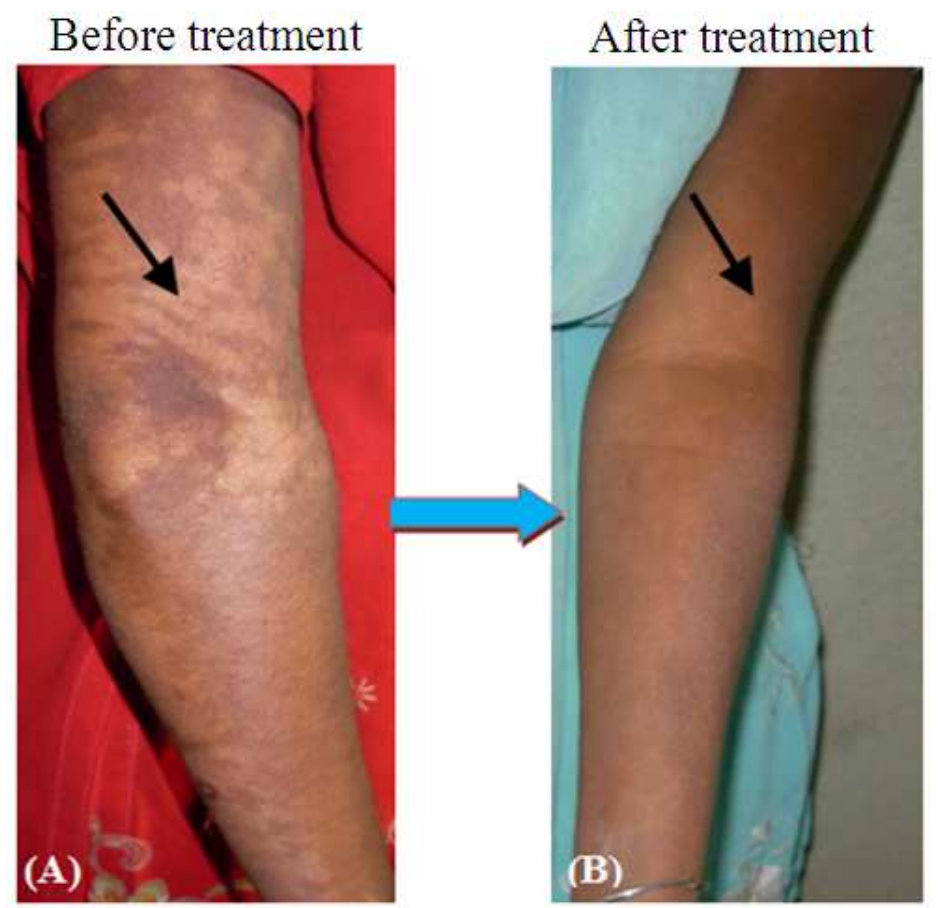

Fig. 1. PKDL presenting as hypopigmented macular lesions on the arm before (A) and after (B) treatment with milterfosine

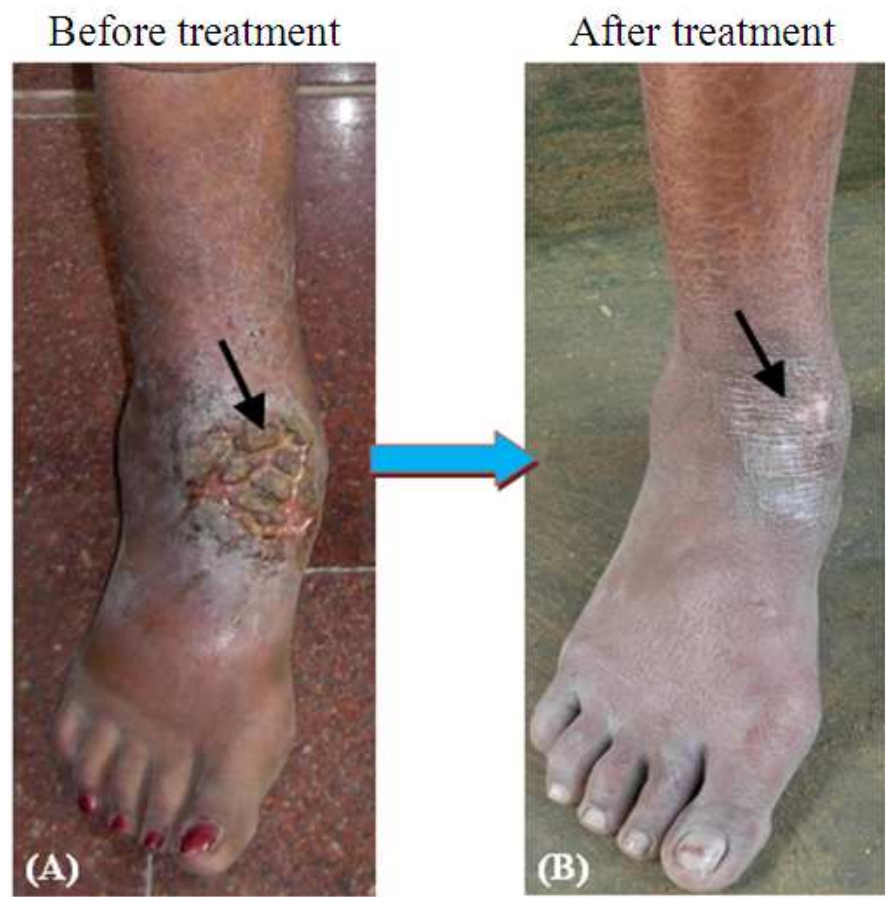

Fig. 2. PKDL presenting as ulcerating lesions on the foot before (A) and after (B) treatment with milterosine 


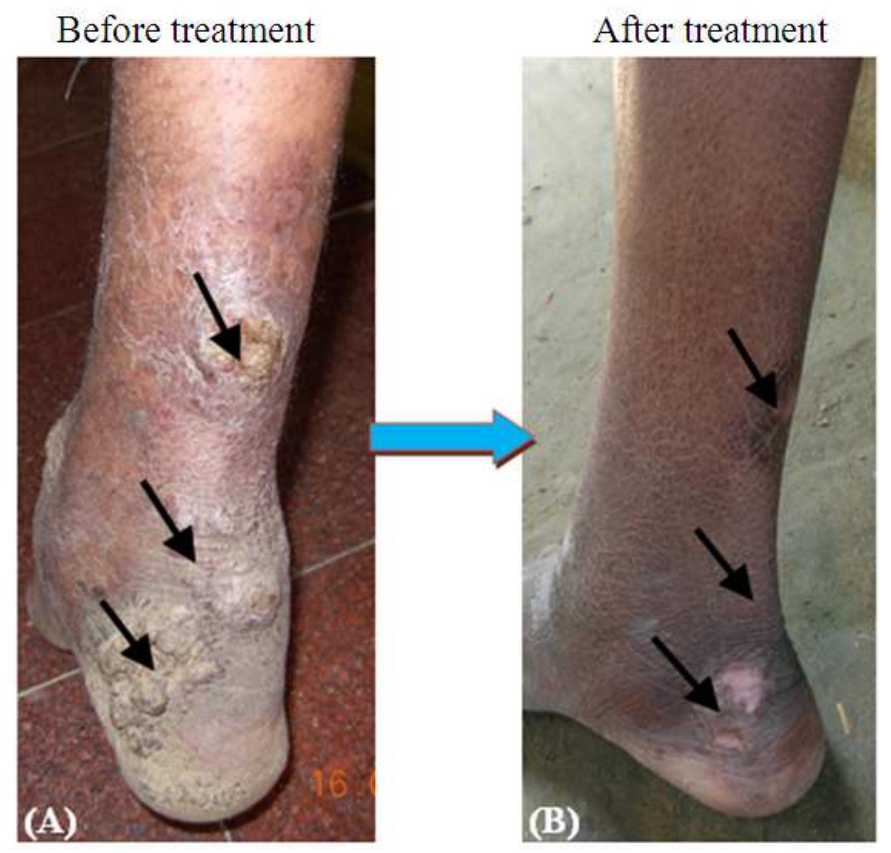

Fig. 3. PKDL presenting as ulcerating lesions on the foot before (A) and after (B) treatment with milterfosine

The patient was further followed-up for one year and no further signs and symptoms of PKDL were observed clinically, parasitologically and by k-DNA nested-PCR assay. Aseptic dressing on alternate days during treatment period was done for the ulcerating lesions.

\section{DISCUSSION}

The diagnosis and the management of PKDL have not been addressed properly in the kala-azar elimination program in the Indian subcontinent, which is very complex. The diagnostic aspect requires clinical expertise, trained pathologist and molecular biologist. PKDL is diagnosed by demonstration of leishmania parasites from the skin snip of the lesions. Besides this PKDL lesions caused by L.donovani can also be confirmed by kinetoplast-DNA nested-PCR assay (Sreenivas et al., 2004). The differential diagnosis includes leprosy (which can be differentiated by loss of pain and temperature over the lesion), fungal lesions like eczematous dermatitis, pityriasis and auto immune diseases like psoriasis (which can be ruled out by a trained dermatologist).

The treatment aspect of PKDL is no less difficult as the duration is very long which can lead to poor compliance and adverse effects of the drugs. Sodium Stibogluconate (SAG), used to be a very potent drug for treatment of VL, has now developed resistance and the cure rate in the endemic districts of Bihar is as low as 52\% (Das et al., 2005). Moreover, its prolonged administration, as required in PKDL, can limit its use particularly because of cardio-toxicity (Thakur and Kumar, 1990). Repeated courses of Amphotericin B have been found to be superior to SAG for treatment of PKDL with a lesser duration (Thakur et al., 1997). However, it requires prolonged intravenous administration in a hospital for treatment as well as monitoring of serum creatinine and potassium levels. In India, miltefosine, the first oral drug, has been introduced as the first-line drug for treatment of VL under elimination programme, but not for PKDL. In a recent study conducted in PKDL, twelve-week treatment with miltefosine was found to have $93 \%$ cure rate, but is contraindicated in female patients of reproductive age group due to possible teratogenic effect (Sundar et al., 2013). However, being a oral drug with minimal side effects, its acceptability at the community level has been found to be superior to the other injectable drugs mentioned above.

Ulceration is not a characteristic feature of Indian and Sudanese PKDL. In a similar case report of PKDL hailing from Bihar presenting with ulcerated chronic paronychia like lesion treatment was given with miltefosine with complete recovery (Jha et al., 2012). 
Miltefosine was also used to successfully treat a pleomorphic PKDL in an Indian patient hailing from Bihar (Pal et al., 2013).

Without exact known mechanism and etiopathogenesis, PKDL disease is not yet defined properly (Thakur et al., 2008). Besides cosmetic significance to the patient more so in females, PKDL is of great epidemiological importance as it serves as a parasite reservoir for VL transmission.

This atypical case report from Bihar, the highest VL endemic state of India, highlights the additional manifestation of skin lesions in the form of ulcer. It seems to be of clinical relevance to treating physicians and dermatologists and warrants to further explore clinical manifestations of PKDL in VL endemic areas.

\section{CONCLUSION}

In conclusion the VL elimination program will not have much impact till PKDL which is of great epidemiological importance as far as the transmission of VL is concerned, is diagnosed early and treated properly.

\section{LIMITATIONS}

Diagnosis and treatment of PKDL is matter of great concern. PKDL is said to be of major epidemiological importance as far as transmission of kala-azar is concerned. Diagnosis requires the joint endeavor of a dermatologist, histopathology's and clinician. This can only be possible in a tertiary care hospital set-up. Simpler diagnostic methods for PKDL have to be searched particularly in the rural field setting. Furthermore short and effective treatment for PKDL has to be developed in order to avoid long term side effects of the antileishmanial drugs. Early diagnosis and treatment of PKDL can lead to eradication of VL from the Indian subcontinent.

\subsection{Conflicts of Interest}

The authors declare that they have no conflicts of interest.

\section{ACKNOWLEDGEMENT}

The researchers wish to thank the sincere efforts of Dr. Vishwa Mohan Katoch, Secretary of Department of Health Research (DHR), Government of India and Director General of Indian Council of Medical Research, New Delhi, India for his continuous guidance in the kalaazar elimination program.

\section{REFERENCES}

Brahmachari, U.N. 1992. A new form of cutaneous leishmaniasis, dermal leishmanoid. Ind. Med. Gaz, 57: 125-127.

Das, V.N, A. Ranjan, S. Bimal and N.A. Siddique, 2005. Magnitude of unresponsiveness to sodium stibogluconate in the treatment of visceral leishmaniasis in Bihar. Nat. Med. J. Ind., 18: 131133.

Das, V.N., A. Ranjan and K. Pandey, 2012. Clinical epidemiologic profile of a cohort of post-kala-azar dermal leishmaniasis patients in Bihar, India. Am. J. Trop. Med. Hyg., 86: 959-961. DOI: 10.4269/ajtmh.2012.11-0467

Jha, A.K., V. Anand, S.K. Mallik and A. Kumar, 2012. Post Kala Azar Dermal Leishmaniasis (PKDL) presenting with ulcerated chronic paronychia like lesion. Kathmandu Univ. Med. J., 10: 87-90.

Pal, D., A. Naskar and M.K. Ghosh, 2013. A case of miltefosine responsive pleomorphic post kala-azar dermal leishmaniasis. Br. J. Infect Dis., 17: 610-612. DOI: 10.1016/j.bjid.2012.12.011

Salotra, P., G. Sreenivas, V Ramesh and S. Sundar, 2001. A simple and sensitive test for field diagnosis of post kala-azar dermal leishmaniasis. Br. J. Dermatol., 4: 630-632. DOI: 10.1046/j.13652133.2001.04434.x

Sreenivas, G., N.A. Ansari, J. Kataria and P. Salotra, 2004. Nested PCR assay for detection of Leishmania donovani in slit aspirates from postkala-azar dermal leishmaniasis lesions. J. Clin Microbiol., 4: 1777-1778. DOI: 10.1128/JCM.42.4.1777-1778.2004

Sundar, S., P. Sinha and T.K. Jha, 2013. Oral miltefosine for Indian post-kala-azar dermal leishmaniasis: A randomised trial. Trop. Med. Int. Health, 18: 96-100. DOI: $10.1111 /$ tmi.12015

Thakur, C.P. and K. Kumar, 1990. Efficacy of prolonged therapy with stibogluconate in post kala-azar dermal leishmaniasis. Ind. J. Med. Res., 91: 144-148.

Thakur, C.P., A. Kumar, G. Mitra, S. Thakur and P.K. Sinha et al., 2008. Impact of amphotericin-B in the treatment of kala-azar on the incidence of PKDL in Bihar, India. Ind. J. Med. Res., 128: 38-44. PMID: 18820357 
Thakur, C.P., S. Narain and N. Kumar, 1997. Amphotericin B is superior to sodium antimony gluconate in the treatment of Indian post kala-azar dermal leishmaniasis. Ann. Trop Med. Parasitol., 91: 611-616. DOI: 10.1080/00034989760707

Verma, N., D. Singh and K. Pandey, 2013. Comparative evaluation of PCR and imprint smear microscopy of skin biopsy in diagnosis of macular, papular and mixed papulo-nodular lesions of PKDL. J. Clin Microbiol., 51: 4217-4219. DOI: 10.1128/JCM.01482-13
Zijlstra, E.E., A.M. Musa and E.A. Khalil, 2003. Postkala-azar dermal leishmaniasis. Lancet Infect Dis., 3: 87-98. DOI: 10.1016/S1473-3099(03)00517-6

Zijlstra, E.E., E.A. Khalil, P.A. Kager and A.M. ElHassan, 2000. Post-kala-azar dermal leishmaniasis in the Sudan: Clinical presentation and differential diagnosis. Br. J. Dermatol., 43: 136-143. DOI: 10.1046/j.1365-2133.2000.03603.x 